\title{
El papel del objeto en el aprendizaje del movimiento básico del segundo movimiento de 'Rosas Danst Rosas' de Anne Teresa de Keersmaeker
}

Sheyna Teixeira Queiroz

Recebido em: 21/10/2018

Aprovado em: 06/11/2019

DOI: $10.5965 / 2358092521222019165$

1 Graduada em Dança pela UFRJ. Mestranda em Dança/Práticas Performativas pela Universitè de Lille, França. E-mail: sheyntq@icloud.com 


\section{RESUMEN}

Este artículo es tema de un estudio práctico y teórico sobre la utilización de objetos (palo, balón, silla, etc.) en el aprendizaje de una coreografía a través de los principios de la educación somática. La metodología se basa en la descripción de la experiencia corporal y en las sensaciones vividas. Dos registros de la performance en vídeo se utilizan como referencias visuales y artículos, libros y notas de intención nos ayudan a discusiones teóricas.

Palabras-clave: danza; educación somática; aprendizaje.

\section{ABSTRACT}

This article consists of studying in practice and in theory the use of objects (stick, ball, chair, etc.) in the learning of a choreography according to the principles of somatic education. The methodology is based on the description of the body experience and on what sensations come into play... Two video performance recordings are used as visual references, and articles, books, and notes of intent help us in the theoretical discussions.

Keywords: dance; somatic education; learning.

\section{INTRODUCCCION}

Se escribió este artículo durante el programa de Maestría en Danza y Prácticas Performativas de la Universidad de Lille SHS en la disciplina de "Enfoque Crítico de Arte Contemporáneo 
- Seminario: Cuerpo, Danza, Performance2" dirigido por Laetitia Doat ${ }^{3}$.

El tema de estudio es el uso de objetos (palo, balón, silla etc.) en el proceso de aprendizaje de la danza-escritura coreográfica, más concretamente en el movimiento básico de la segunda secuencia del espectáculo "Rosas danst Rosas" de la Compañía Belga, "Rosas"4, de la coreógrafa Anne Teresa de Keersmaeker5.

2 El seminario tiene como objetivo vincular una práctica con el trabajo de investigación académica; Cómo describir una práctica; Poner palabras en los ejercicios (improvisaciones y escritura); Cómo describir una experiencia sensible - desde y sobre la práctica de la danza; Cómo hacer protocolos de sesión o clases; Descubrir cómo la práctica cambia la mirada con relación al corpus de la investigación, es decir, la práctica en relación con el protocolo de investigación. Información dada oralmente por la Sra. Doat en la segunda clase del Seminario: Cuerpo, danza, performance el 02/03/2017 - Estudio de Danza de la Universidad de Lille 3: Semestre 2017.1.

3 Después de terminar sus estudios superiores en literatura, obtuvo un título en danza contemporánea y clásica en el Conservatorio Regional de Toulouse, Laetitia Doat se matriculó en una licenciatura en el departamento de danza de la Universidad de París 8 en 2002. Ella hizo su doctorado en 2013. Participa regularmente en los talleres de doctorado ofrecidos en el Centro Nacional de Danza, es miembro de la Asociación de Investigadores de Danza. También fue miembro del consejo editorial de la revista Repères, durante cuatro años (2006-2009). Más información en: http://www.danse.univ-paris8.fr/chercheur.php?cc_id=3\&amp;ch_id=52

\section{4 https://www.rosas.be}

5 Bailarina y coreógrafa belga. A la edad de diez años, estudió en la escuela de danza de Bruselas presidida por Maurice Béjart y luego la famosa Escuela New York Tisch School of the Arts le abrió sus puertas. Allí, ella fue introducida a la danza americana posmoderna. Rosas nace de su impulso, una empresa atípica que circunnavega el mundo desde los años 80. Desde Estados Unidos, Nueva Zelanda y Europa. En la década de los noventa, inicia una colaboración con el Teatro La Monnaie de Bruselas, donde presenta a Stella, Elena, Aria, que conduce a la creación de P.a.r.t.s, una escuela que revela la capacidad de la coreógrafa de dirigir una compañía de baile y dirigirlo a un aprendizaje tan intelectual como físico. Su creación original Soirée Steve Reich de Steve Reich en el Théâtre de la Ville en 2007 rinde homenaje a la música minimalista del músico. Anne Teresa de Keersmaeker ha despertado emoción desde los años 80 y participa en la renovación de la danza posmoderna con discernimiento. Texto disponible: https://www.forum-meyrin.ch/sites/default/files/private/dp_rosas_danst_rosas. 
Nos interrogamos en el uso de objetos desde una perspectiva de educación somática donde el aprendizaje se basa en la conciencia corporal y la propiocepción (el cuerpo en el espacio). La metodología sigue un estudio teórico que se refiere a textos y artículos sobre educación somática así como el espectáculo propuesto. Por otro lado, seguimos con la descripción de una práctica que nos permite expresar los sentimientos y sensaciones, es decir, hablamos de una teoría "experimentada" o "vivenciada".

"Rosas danst Rosas" es un espectáculo creado en 1983 por Anne Teresa de Keersmaeker en el Teatro Balsamine de Bruselas con música de Thierry de Mey $^{6}$ y Peter Vermeersch 7 . Se utilizan dos herramientas visuales: el video-registro, filmado en junio de 1995 de la performance en Lunatheater y la video-danza filmada en 1997 por Thierry de Mey.

La sesión descrita en este trabajo tuvo lugar el día 2 de marzo de 2017, de las 9 de la mañana a las 12 de la noche en el "Studio de Danse de la Universidad de Lille SHS" bajo la dirección de la Sra. Laetitia Doat para los estudiantes de la Maestría en Danza / Prácticas Performativas.

pdf - Acceso en 27/08/2019.

6 Thierry De Mey es un compositor y cineasta. Sus creaciones implican la interacción de música, danza, video y procesos interactivos. Se han presentado en eventos como las Bienales de Venecia, Lyon y muchos museos. Su obra ha sido galardonada con premios nacionales e internacionales (Premio Bessie, Eve du Spectacle, Foro de Compositores de la Unesco, FIPA...). Texto completo disponible: https://www.forum-meyrin.ch/sites/default/files/private/dp_rosas_danst_rosas. pdf Acceso en 27/08/2019.

7 Peter Vermeersch, fundador de Flat Earth Society (FES), es compositor, clarinetista, saxofonista y productor de bandas de rock. La singular música de FES va desde la pura improvisación hasta la música basada en partituras y alegremente atraviesa los diferentes géneros. FES no sólo es experimentado en el campo del jazz, sino que también está abierto a otras formas artísticas como el teatro o el cine. Se puede citar a Larf, una actuación de y con Josse De Pauw, The score for Minoes, de Vincent Bal y su proyecto más reciente, De Oesterprinses, por Ernst Lubitsch. Texto disponible: https://www.forum- meyrin.ch/sites/default/files/private/dp_rosas_danst_rosas. pdf Acceso en 27/08/2019. 
EL PAPEL DEL OBJETO EN EL APRENDIZAJE DEL MOVIMIENTO BÁSICO DEL SEGUNDO MOVIMIENTO DE 'ROSAS DANST ROSAS' DE ANNE TERESA DE KEERSMAEKER

Entonces, ¿cómo estos objetos pueden ayudar somáticamente al aprendizaje de la segunda secuencia de "Rosas danst Rosas"?

\section{OBJETO, PROPRIOCEPCIÓN Y MULTISENSO- RIALIDAD}

\section{DESCRIPCIÓN DE LA SESIÓN: MOVIMIENTO APRENDIZAJE Y OBJETOS UTILIZADOS}

En la sesión usamos: 1 palo, 2 sillas y 2 pequeñas pelotas llenas de agua (cada persona). Los pies llevan zapatos - sin tacones. Al principio había una silla: uno se sienta de tal manera que los isquiones están atascados en la silla y los pies fluyen desde el suelo; una pequeña pelota de agua entre los tobillos y el otro entre las rodillas; el palo en las manos a nivel del ombligo (las manos están en el centro para que se separen como la distancia entre los hombros). Los brazos siguen la misma dirección haciendo un ángulo de 90 grados en los codos. La palma de la mano está hacia abajo.

Las indicaciones del comienzo son hacer el mínimo esfuerzo posible en el movimiento y que sea el palo llevado por el brazo y no lo contrario, de tal modo que no haya dolor ni fatiga cuando se hace. Las pequeñas pelotas de agua entre las rodillas y entre los tobillos dan una referencia propioceptiva de la distancia entre ellos y la alineación general del cuerpo.

El palo pasó un poco más alto, al nivel de los hombros. En secuencia, fueron los codos a nivel de los hombros (una vez los codos forman un ángulo de 90 grados) y el palo delante de la cabeza. Entonces el palo se levanta hacia arriba y hacia atrás, donde permanece a nivel de lo alto de la cabeza.

Dejamos un poco el palo y las pelotas de agua al lado para aprender los movimientos de las piernas: uno comienza con la apertura de las piernas en segunda posición paralela con la parte superior del cuerpo bien erguido; las piernas y el tronco siguen la misma dirección (en este movimiento las piernas for- 
man una cuarta posición paralela donde la pierna izquierda está doblada a 90 grados y la pierna derecha permanece detrás en el ángulo más abierto). Se vuelve a la posición del principio con todo el movimiento inverso.

Para aprender los movimientos de los brazos y de la cabeza, se toma de nuevo el palo y una silla más (uno está sentado en la silla a la derecha): el brazo izquierdo se posa en la parte posterior de la silla a la izquierda y el brazo derecho se posa sobre el asiento; El tronco baja y luego vuelve a la posición inicial con las manos cruzadas y colocadas sobre las piernas. Los brazos van directamente a nivel del ombligo, cuando vuelven, el tronco baja; La mano derecha viene hacia la cabeza y acaricia el cabello, mientras la mano izquierda descansa sobre las piernas. A continuación, ambas manos se unen al nivel del cuello, cuando los codos siguen unidos también; Las manos acarician el cabello al girar la cabeza; la mano derecha toca el seno derecho; El tronco baja hasta el brazo derecho que se alarga hacia el suelo; todo el cuerpo vuelve a la posición del principio. Ambos brazos van hacia las rodillas (en las piernas), el tronco sigue el movimiento hacia adelante; La cabeza baja y entra para mirar el horizonte delante, hasta cuando la mano derecha cuelga la cabeza que mira (con el codo en la rodilla derecha). Todo el tronco baja y vuelve a la posición inicial. Los pies y las piernas se abren en la segunda posición paralela; ambos brazos van a la izquierda hasta las piernas abiertas en la cuarta posición paralela (como la descripción del ejercicio); los brazos vuelven y entran entre las dos piernas que están cerradas; La cabeza mira hacia arriba y todo el cuerpo vuelve a la posición inicial. El cuerpo se levanta (de pie) con los brazos que acompañan el movimiento hasta llegar a la cabeza y acariciar el cabello; Los brazos pierden peso como si renunciaran a algo y el cuerpo vuelve a sentarse con el tronco en forma de una " $\mathrm{C}$ ".

Se repitió varias veces, sin música y con música. Tres palabras fueron escritas para describir la experiencia, tres adjetivos y tres verbos. Así terminó la sesión. 


\section{APRENDIZAJE A TRAVÉS DE LA EDUCACIÓN MULTISENSORIAL E INTERDISCIPLINARIA}

Recordémonos que la sesión que dirigió la Sra. Doat fue multisensorial: ella nos dio varias herramientas directa o indirectamente (objetos, movimientos, video, música, información relacionada con el reconocimiento del cuerpo y su posición en el espacio, información sensible, física y psicológica en relación con la autoconciencia etc.). Sus herramientas hacen posible llegar a la mayoría de los estudiantes y hacerlos entender y aprender, tener algunas propiedades interdisciplinarias. Queiroz (2015), nos da la referencia de cómo un vídeo danza puede llegar al público a través de la interdisciplinariedad y cómo la interdisciplinariedad llega a la pluralidad de diferentes inteligencias:

El proceso educativo es continuo, debe ser persistente e interdisciplinario para llegar a todos los individuos en sus preferencias sensoriales y experiencias de vida. Una video danza puede criticar y desarrollar conceptos educativos para captar diferentes sentidos sensoriales, en relación con la activación de diferentes inteligencias humanas: lógico-matemáticas, confrontación de elementos y conceptos abstractos; lengua, el uso de la poesía escrita y oral; música por elección y búsqueda rítmica y timbre, y composición del ambiente y sonidos musicales; espacio, para recrear el espacio a través de ángulos y cortes cinematográficos; cuerpo-kinestésico, presencia y diálogo con la danza; intrapersonal, la perspectiva de poner y ser reconocido en ciertas situaciones y/o partes del video; La comprensión interpersonal de que la contaminación y la degradación son la causa y la consecuencia de la acción de todos; naturalista, la capacidad de entender que el mundo es el conjunto de seres de un solo ecosistema; existencial, la capacidad de comprender el lado filosófico, metafísico y espiritual presente en la relación entre el hombre, la naturaleza y el medio ambiente. (QUEIROZ, 2015, p. 169-170, trad. nuestra).

Estas indicaciones nos hacen entender la totalidad de una sesión de aprendizaje y la posibilidad de acercarnos más fácilmente a los estudiantes y que esta conciencia puede aplicarse en todo el ámbito de la enseñanza. 


\section{‘ROSAS DANST ROSAS' Y APRENDIZAJE SO- MÁTICO}

Ahora presentaremos al lector la obra coreográfica "Rosas danst Rosas", la vídeo danza y el espectáculo. También debemos presentar los conceptos del empleo del término "educación somática" y las sensaciones con relación con la clase presentada antes (en el momento en que se habla de sentimiento, se utilizará el "yo" por una simple razón, no podemos decir "sentir" en plural cuando es una percepción personal).

Utilizaremos el concepto de educación somática de Joly (2006, p.23, trad. nuestra): "La educación somática es el campo disciplinario de un conjunto de métodos cuyo objeto es el aprendizaje de la conciencia del cuerpo en movimiento en el medio ambiente".

Este concepto nos da una comprensión de la amplitud de las posibilidades de las técnicas en la educación somática. Además, entendemos que el objetivo es siempre el mismo: la conciencia del cuerpo y su relación con el movimiento, con otros cuerpos y con el espacio.

La experiencia de aprender una secuencia de danza marcada por el ritmo musical, con el tiempo específico, nos obliga a memorizar cada movimiento.

Tuve cierta dificultad en relación con esta memorización, lo comenté con la señora Doat, que confirmó la necesidad de repetir hasta que la coreografía se entendiera bien, e incluso, que el cuerpo lo sintiera bien. Esta comprensión y aprehensión del movimiento es un proceso mental, físico, corporal y emocional:

Como ejemplo, la memoria misma es un acontecimiento de todo el organismo, y no sólo está localizada en alguna parte del cerebro. La memoria se refiere al sentido del yo como experimentado en la imagen del cuerpo que es parte de lo que se recuerda (Rosenfield, 1996 apud JOLY 1996). [...] El pensamiento, las emociones, las sensaciones, la abstracción se experimentan en la musculatura, la frecuencia cardíaca, la respiración, la postura, incluso si desde el exterior el cuerpo muestra poco movimiento en el espacio. (JOLY, 2006, p. 22, trad. nuestra). 
En el ejercicio del palo, la señora Doat hizo indicaciones a las personas que tenían algún desequilibrio con la línea recta que debían hacer el objeto palo. Traté de mirarme a mí misma para saber que era yo quien no lo hacía bien, pero no podía sentir ni siquiera mirarme a mí misma.

Joly (2006, p. 21, trad. nuestra) nos da una razón:

No podemos estar muy lejos de la definición de la neurosis de Karen Horney: cuando, a sabiendas de nosotros, hacemos lo contrario de lo que pensamos. [...] Schilder (1968) apud JOLY 2006 ya había anunciado: 'la imagen del cuerpo nunca es una estructura completa; no es estático; siempre hay perturbaciones'.

Así, conocemos la necesidad de crear esta imagen del cuerpo antes de comenzar a aprender el movimiento. La condición pedagógica de la educación somática se confirma para Joly (2006, p. 23, trad. nuestra):

Es este proyecto de educación somática el que emprendo con mis clientes o pacientes, a los que prefiero llamar 'estudiantes', ya que de hecho es un marco pedagógico que está aquí: aprender la conciencia del cuerpo, completa y ajusta la imagen de sí mismo, desarrolla la conciencia.

Es cierto que cuando se mira la vídeo danza o el espectáculo "Rosas danst Rosas", se piensa que las transfiguraciones de los gestos cotidianos son muy expresivos y teatrales, la dramaturgia es muy femenina con referencias a los movimientos de las mujeres o incluso se nos fijamos en los trajes: "faldas cortas y camisas blancas, calcetines de tobillo y botas barnizadas, las cuatro mujeres juegan sobre los ritmos musicales, pero también sobre las gotas de tensión"8. Mirando hacia atrás una segunda vez, percibimos que la repetición de los movimientos danzados da un contexto dinámico, fuerte y firme, las sensaciones de fati-

8 Nota de intención escrita por Marianne Van Kerkhoven (1998), disponible en: https://www.forum-meyrin.ch/sites/default/files/private/dp_rosas_danst_rosas. pdf. Acceso en 27/08/2019. 
ga y la forma de volver al inicio como en la rutina de la vida cotidiana: "construida en cuatro pasos rítmicos por la respiración o por el sonido de la música también repetitiva de Thierry de Mey y Peter Vermeersch, esta obra entremezcla el gesto y la abstracción diarios"9. Todavía en la nota de intención del espectáculo, Kerkhoven (1998, trad. nuestra) describe:

En Rosas danst Rosas, dos tipos de movimientos se entrelazan. Por un lado, movimientos abstractos, difíciles de calificar, por el otro, movimientos más concretos, reconocibles: la mano que alisa el cabello, que rectifica sosteniendo la blusa, la cabeza que se vuelve abruptamente ... estos gestos que se refieren a los gestos cotidianos tienen un significado directo. El material gestual es continuamente astillado hasta que los bailarines muestran su fatiga: se colocan en el escenario, visiblemente sin aliento y empapado de sudor.

Los ensayos se realizan juntos (los cuatro bailarines al mismo tiempo) o no, mientras se contempla el segundo movimiento del espectáculo en este principio de creación coreográfica. En el vídeo danza podemos escuchar el sonido de la respiración, el movimiento de la danza que corta el aire y el tacto entre las partes del cuerpo. Además, cuando se conoce la escritura coreográfica, realmente se comprende la necesidad de expresar todo el sonido, como si él pudiera decir lo que no se puede con la voz; como si los gestos quisiesen gritar.

Sobre la repetición en el trabajo la nota de intención dice:

[...] De Keersmaeker nunca intenta alcanzar la perfección absoluta en sus coreografías: no requiere que sus bailarines actúen sin ninguno error los movimientos simultáneos. Es por eso que las representaciones de Rosas siempre dan testimonio de esta expresividad y de esta humanidad tan específica. Kerkhoven (1998, trad. nuestra)

9 Ídem. 
Aunque me parezca absoluta en el tiempo y en la música, la escritura física es demasiada precisa, casi como un ballet clásico, excepto los movimientos que se basan en los gestos cotidianos, pero no puedo decir nada en el proceso de creación. También parece ser la diferencia entre el punto de vista del creador y el punto de vista del público.

Después de la sesión, describiré el trabajo como: pulsando rápida y tristemente, con sensualidad y feminidad; la belleza está presente en el contexto general de la expresión y la contradicción, la belleza en el sentido estético de las sensaciones y sentimientos traídos por el arte, es decir, el disfrute estético. Voluntad y cansancio, los binomios complementarios que componen mi descripción final.

\section{CONCLUSIÓN}

Este trabajo es un ensayo que cuestiona las relaciones entre prácticas de danza y escritura académica de investigadores-bailarines-coreógrafos.

En relación con la problemática del inicio, se responderá a la pregunta inicial: el objeto utilizado en la práctica de enseñanza-aprendizaje de una secuencia de movimiento coreográfico específico es efectivo dentro de la pedagogía somática en la danza.

Nuestra respuesta se demuestra con los argumentos y conceptos presentados en el corpus de la investigación. Sobre todo, uno siente su relevancia cuando, unos días después de la sesión, pudo describir la práctica y la danza, es decir, que se alcanzó el objetivo de la sesión.

Además, el análisis de Guisgand (2002) sobre la obra en cuestión, nos da otra perspectiva problemática en relación con nuestra primera cuestión. El autor propone un análisis de las bases corporales de un estilo coreográfico. Entonces, nos preguntamos: ¿ Es efectiva la contribución somática como método para aprender otros estilos coreográficos? Para un estilo coreográfico específico ¿existe una metodología específica de aprendizaje 
también? ¿Es el estilo personal o metodológico (en relación con el profesor y una técnica o metodología específica)?

Una vez más, después de leer el análisis de Guisgand (2002) sobre el segundo movimiento de "Rosas danst Rosas", me pregunto sobre el método de descripción: ¿cualitativa o cuantitativa? ¿Subjetivo u objetivo? ¿Libre o cronológico? ¿Simbólico o metafórico? ¿Personal o impersonal?

Así presentamos una serie de preguntas que va más allá de nuestra primera mirada lo que prueba también la riqueza de la sesión y el trabajo elegido para el análisis. 


\section{REFERENCIAS}

ACOSTA, A. Dança e Cinema: algumas aproximações. Revista Gambiarra. Rio de Janeiro, n.4, p. 22-28, 2012.

GUISGAND, P. Vers un modèle d'analyse fonctionnelle en danse: Rosas danst Rosas d'Anne Teresa de Keersmaeker. Revista Deméter. Lille n.12/2002, p.1-11, 2002.

JOLY, Y. L'image du corps et la conscience de soi. Revista Psychologie Québec, Quebec, n.05/2006, p. 21-23, 2006.

KERKHOVEN, M.V. Note d'intention "Rosas danst Rosas". Forum Théâtre Meyrin, 2011. Disponible en: https://www.forummeyrin.ch/sites/default/files/private/dp_rosas_danst_rosas.pdf. Acceso en 27/08/2019

QUEIROZ, S. T. A poética da vida em poluição: diálogos interdisciplinares de arte, dança \& sustentabilidade. Michoacán: CRAM Ediciones, 2015. 